\title{
A Computational Study of Residual KPP Front Speeds in Time-Periodic Cellular Flows in the Small Diffusion Limit
}

\author{
Penghe $\mathrm{Zu}$, Long Chen, Jack Xin *
}

\begin{abstract}
The minimal speeds $\left(c^{*}\right)$ of the Kolmogorov-Petrovsky-Piskunov (KPP) fronts at small diffusion $(\epsilon \ll 1)$ in a class of time-periodic cellular flows with chaotic streamlines is investigated in this paper. The variational principle of $c^{*}$ reduces the computation to that of a principle eigenvalue problem on a periodic domain of a linear advection-diffusion operator with space-time periodic coefficients and small diffusion. To solve the advection dominated time-dependent eigenvalue problem efficiently over large time, a combination of spectral methods and finite element, as well as the associated fast solvers, are utilized to accelerate computation. In contrast to the scaling $c^{*}=\mathcal{O}\left(\epsilon^{1 / 4}\right)$ in steady cellular flows, a new relation $c^{*}=\mathcal{O}(1)$ as $\epsilon \ll 1$ is revealed in the time-periodic cellular flows due to the presence of chaotic streamlines. Residual propagation speed emerges from the Lagrangian chaos which is quantified as a sub-diffusion process.
\end{abstract}

Keywords: KPP front speeds, time periodic cellular flows, chaotic streamlines, sub-diffusion, residual front speeds.

PACS: 42.25.Dd, 02.50.Fz, 05.10.-a, 02.60.Cb

* Department of Mathematics, UC Irvine, Irvine, CA 92697, USA. Corresponding author: Penghe Zu. Phone number: 9493784420.

Emails: (pzu,chenlong,jxin)@math.uci.edu. 


\section{Introduction}

Front propagation in complex fluid flows arises in many scientific areas such as combustion [23,28], population growth of ecological communities (plankton) in the ocean [1], and reactive chemical front in liquids [22,29]. An interesting problem is the front speed enhancement in time dependent fluid flows with chaotic streamlines and random media [22, 29].

In this paper, we shall consider a two dimensional time dependent cellular flow:

$$
(\cos (2 \pi y)+\theta \sin (2 \pi y) \cos (t), \cos (2 \pi x)+\theta \sin (2 \pi x) \cos (t)), \quad \theta \in(0,1],
$$

whose steady part $(\cos (2 \pi y), \cos (2 \pi x))$ is subject to time periodic perturbation that causes transition to Lagrangian chaos. Chaotic behavior of a similar flow field is qualitatively analyzed by formal dynamical system methods in [8]. The enhanced residual diffusion in (1.1) is observed numerically in [6]. The enhanced diffusion and propagation speeds in steady cellular flows with ordered streamlines and their extensions have been extensively studied $[2,3,9,12,14-16$, $21,24,26,30,31]$ among others. We shall take a statistical look at the chaotic streamlines of (1.1) in terms of the scaling of the mean square displacements from random initial data, and quantify the Lagrangian chaos of (1.1) as a subdiffusion process.

Consider then the advection-reaction-diffusion equation:

$$
\partial_{t} u=\epsilon \triangle u+\vec{B}(x, t) \cdot \nabla u+\frac{1}{\tau} f(u), \quad x \in \mathbb{R}^{2}, t>0,
$$

where $f(u)=u(1-u)$ is the Kolmogorov-Petrovsky-Piskunov (KPP) nonlinearity, $\epsilon$ is the molecular diffusion parameter, $\tau$ is the reaction rate and $\vec{B}(x, t)$ is a space-time periodic, mean zero, and incompressible flow field such as (1.1). If the initial data for $u$ is nonnegative and compactly supported, the large time behavior of $u$ is an outward propagating front, with speed $c^{*}=c^{*}(\vec{e})$ in the direction $\vec{e}=\langle 1,0\rangle$. The variational principle of $c^{*}$ is [19]:

$$
c^{*}=\inf _{\lambda>0} \frac{\mu(\lambda)}{\lambda},
$$

where $\mu(\lambda)$ is the principle eigenvalue of the periodic-parabolic operator:

$$
L^{\lambda} \Phi=\epsilon \triangle \Phi+(\vec{B}+2 \lambda \vec{e}) \cdot \nabla \Phi+\left(\epsilon \lambda^{2}+\lambda \vec{B} \cdot \vec{e}+\tau^{-1} f^{\prime}(0)\right) \Phi-\Phi_{t},
$$

on the space-time periodic cell $\Omega$. The principle eigenvalue $\mu(\lambda)$ can be computed by solving the following evolution problem:

$$
\begin{aligned}
& w_{t}=\epsilon \Delta w+(2 \epsilon \lambda \vec{e}+\vec{B}(x, t)) \cdot \nabla w+\left(-C_{M}+\epsilon \lambda^{2}+\lambda \vec{e} \cdot \vec{B}(x, t)+\frac{1}{\tau} f^{\prime}(0)\right) w \\
& w(x, 0)=1 .
\end{aligned}
$$


Here the constant $C_{M}=\epsilon \lambda^{2}+\lambda\|\vec{B}\|_{\infty}+\left\|\frac{1}{\tau} f^{\prime}(0)\right\|_{\infty}$, so that $w$ remains positive and bounded by one. The $\mu(\lambda)$ is then given by:

$$
\mu(\lambda)=C_{M}+\lim _{t \rightarrow \infty} \frac{1}{t} \ln \int_{\Omega} w(x, t) d x
$$

The number $\mu(\lambda)$ is also the principle Lyapunov exponent of the parabolic equation (1.5), and the formula (1.6) extends to the more general case when $\vec{B}$ is a stationary ergodic field [18]. The limit then holds almost surely and $\mu$ is deterministic [18]. KPP fronts are examples of the so called "pulled fronts" [25] because their speed is determined by the behavior of the solution far beyond the front interface, in the region where the solution is close to zero (the unstable equilibrium). The minimal speed of the planar wave solution of the linearized equation near the unstable equilibrium gives (1.3), also known as the marginal stability criterion $[25,29]$.

Equation (1.5) and formula (1.6) will be discretized for approximating $\mu$ at sufficiently large time for a range of $\lambda$ values. The minimal point of $\mu(\lambda) / \lambda$ is searched by the Golden-section algorithm. At each new search of $\lambda$, the large time solution of (1.5) is computed. Because $\epsilon$ is small, (1.5) is advection dominated. To this end, upwinding type finite element methods (EAFE [32]) and semi-implicit method are utilized. Spectral method (SM) has high accuracy but the computation is slower because it takes small time step due to the restriction of stability. On the other hand, EAFE can be solved faster using higher accuracy time step with implicit discretization. Based on the stability analysis of upwinding Crank-Nicolson method, we shall see that this scheme is unconditional stable. The fact that EAFE is derived from upwinding scheme shows the similarity between EAFE and upwinding scheme and they will share the same stability analysis. On the other hand, in order to obtain sufficient time accuracy on $\mathrm{SM}$, the time step of $d t=10^{-8}$ is used to capture the limit of front speed. The convergence of the limit with several different time steps indicates that this limit is accurate. In summary, our strategy to obtain the minimal point is to quickly narrow down the search interval by EAFE first, then solve more accurately in a smaller interval by SM without the compromise of accuracy.

The rest of the paper is organized as follows. In section 2, we illustrate the difference of streamline geometry and dynamical properties of steady and unsteady cellular flows. We find that the chaotic streamlines of the time periodic cellular flow (1.1) can be quantified as a sub-diffusive process with distinct $\theta$ dependent scaling exponents. The resulting motion appears ergodic inside the invariant infinite channel domains. In section 3 , we discuss numerical methods for advection-dominated problems such as EAFE, and semi-implicit SM, as well as the stability of these methods and time step constraints. In section 4, we show numerical results of KPP front speeds by a combination of these methods and the existence of residual front speed in the sense that $c^{*}(\epsilon)=O(1)>0$ as $\epsilon \downarrow 0$. In contrast, $c^{*}=O\left(\epsilon^{1 / 4}\right)$ in steady cellular flows [21]. The streamlines of the steady cellular flows are ordered with closed orbits leading to a much slower rate of enhancement for the transport. We also observe the presence of layer and 
circular structures in the generalized eigenfunction $w$ at large time, a reflection of the advection-dominated transport in time periodic cellular flow (1.1). The corresponding energy spectrum of $w$ shows decay towards high frequencies with an intermediate scaling range. In section 5 , we give concluding remarks about our findings.

\section{Properties of Cellular Flows}

In this section, we illustrate the difference of streamline geometry and dynamical properties of steady and unsteady cellular flows. In particular, we quantify the chaotic behavior of the motion along streamlines of (1.1) using the empirical mean square distance inside infinite invariant channels in the direction $(1,1)$, i.e. $E\left[|X(t) \cdot(1,1)|^{2}\right]$, where $X \in \mathbb{R}^{2}$ denotes the particle trajectories in the flow, defined by $\frac{d X(t)}{d t}=\vec{B}(X, t)$. We show computationally that it scales with time as $O\left(t^{p}\right), p \in(0,1)$, for sampled values of $\theta \in(0,1]$.

\subsection{Steady Cellular Flow}

A phase portrait of the steady cellular flow

$$
B_{s}=(\cos (2 \pi y), \cos (2 \pi x)),
$$

with a cell square is presented in Fig. 1. The phase portrait away from the square simply repeats. The streamlines are ordered. The closed orbits form elliptic part of the phase space. The saddles are located at half-integer points $(n, m) / 2, n, m \in \mathbb{Z}$ with connecting separatrices forming hyperbolic part of the phase space. Any particle trajectory is either a closed orbit or a separatrix, hence the motion is bounded inside the square.

\subsection{Unsteady Cellular Flow}

For the unsteady flow

$$
B_{u}=(\cos (2 \pi y)+\theta \sin (2 \pi y) \cos (t), \cos (2 \pi x)+\theta \sin (2 \pi x) \cos (t)),
$$

the invariant manifold consists of lines $y=x+n, n \in \mathbb{Z}$. The flow trajectories are restricted in the channels bounded by two neighboring lines $y=x+n+1$ and $y=x+n$. At $\theta>0$, the flow trajectory extends itself from one cell square to another. The Lagrangian particle undergoes chaotic motion, see Fig. 2 for an illustration. The computation is done by a fourth order symplectic scheme. The local flow direction is colored red. The intensified red region in the middle indicates that the particle spends a lot of time wandering in and out of the cells there. Fig. 3 plots a projected trajectory in the direction $(1,1) / \sqrt{2}$ vs. time. The stochastic feature is visible. 


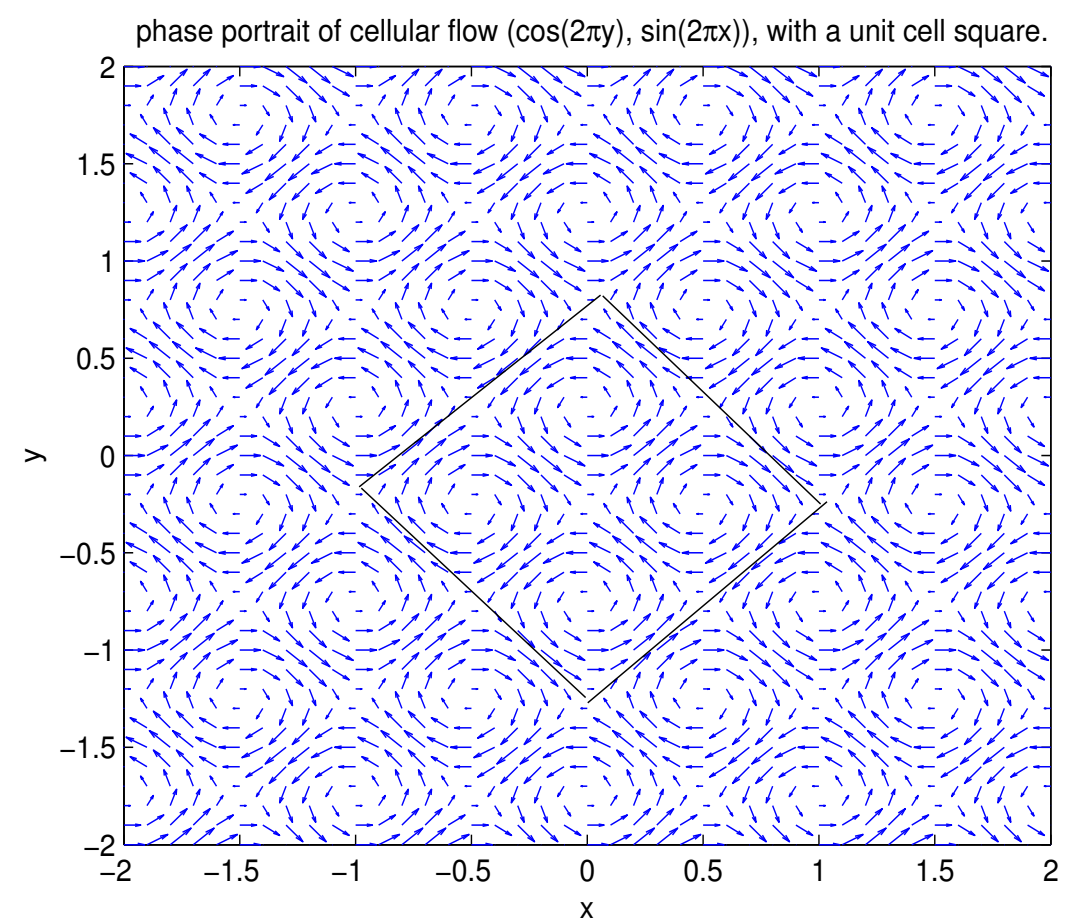

Figure 1: Ordered and localized streamlines of steady cellular flow (2.1) with a cell square containing four vertices. 

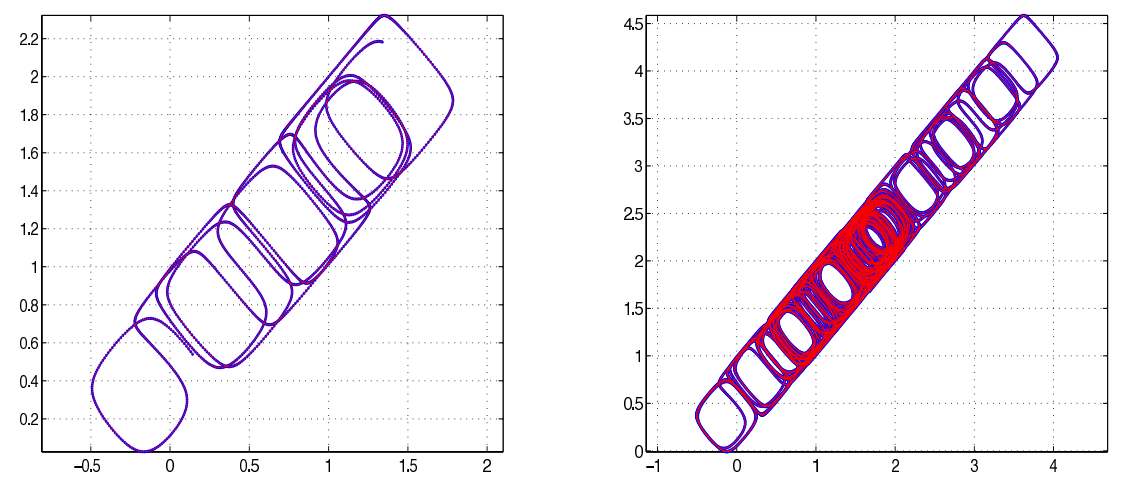

Figure 2: Disordered and extended trajectories in time periodic cellular flow $(2.2)$ over time $[0,20]$ (left) and $[0,200]$ (right) in a channel bounded by invariant lines $y=x+1$ and $y=x$. The local flow direction is colored red.

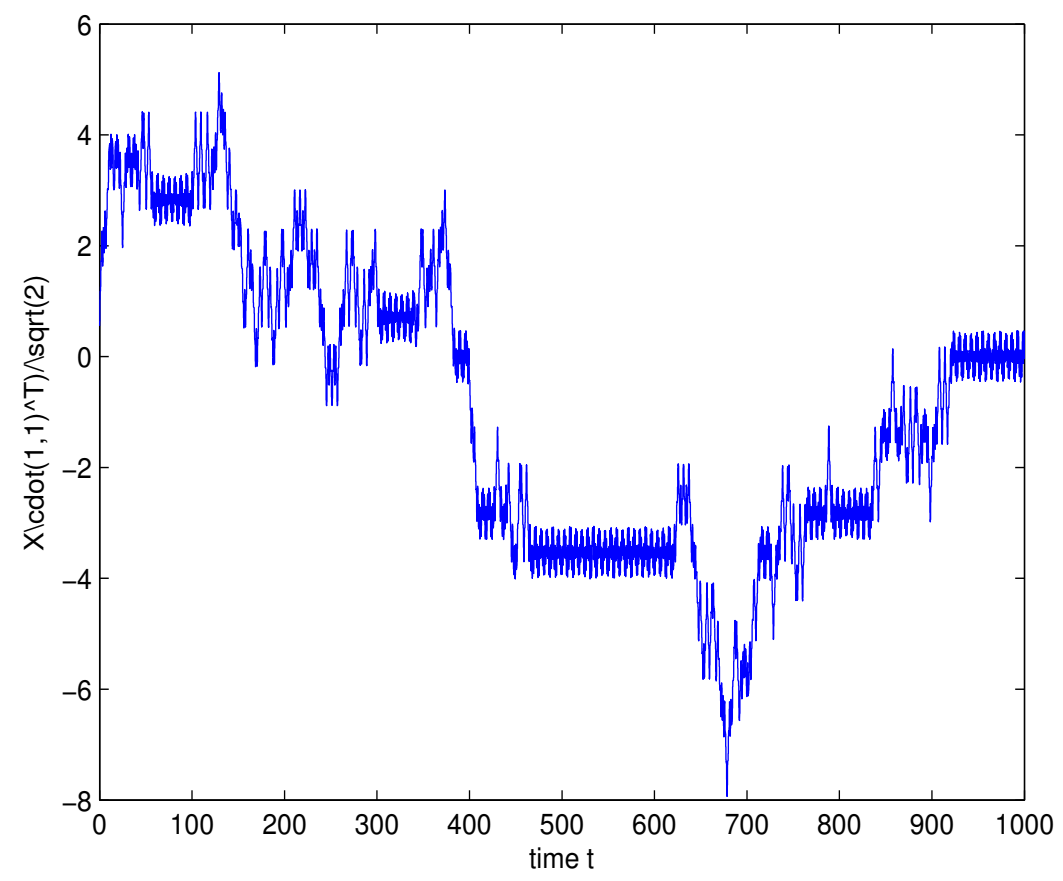

Figure 3: A projected trajectory $X(t) \cdot(1,1) / \sqrt{2}$ in time periodic cellular flow $(2.2), \theta=1$, showing stochastic character. 
To quantify the disorder and draw a connection with diffusion process, we compute $10^{4}$ trajectories $X(t, \omega)$ over time interval $[0,1000]$ with uniformly distributed initial points on a base cell square, where $\omega$ denotes the random samples. We calculate the empirical mean square distance $\left(E\left[|X(t, \omega)|^{2}\right]\right)$ and the projected mean square distance $E\left[(X(t, \omega) \cdot(1,1))^{2}\right]$, and plot them as a function of time on the logarithmic scale to recover scaling laws. For efficiency, the samples are obtained by a 500 node parallel implementation of the standard 4th order Runge-Kutta method. Fig. 4 shows that the mean square distances $E\left[|X(t, \omega)|^{2}\right] \sim O\left(t^{p}\right), p \approx 0.483,0.673$ at $\theta=0.1,0.4$ respectively, hence belonging to the sub-diffusive regime. Similar sub-diffusive behavior can be observed in Fig. 5 where the projected mean square distance scales like $E\left[(X(t, \omega) \cdot(1,1))^{2}\right] \sim O\left(t^{q}\right), q \approx 0.605,0.786$ at $\theta=0.1,0.4$.
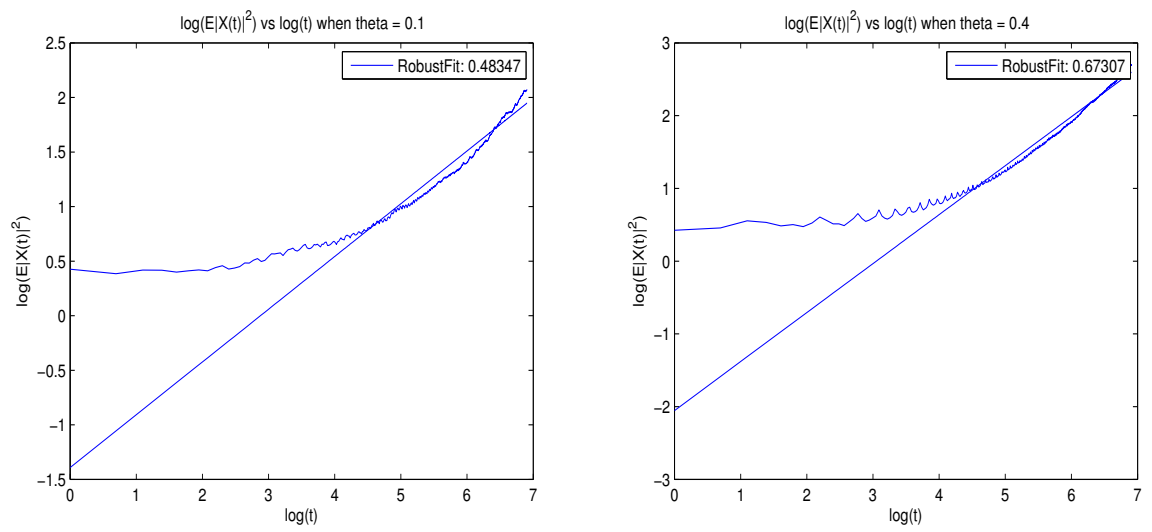

Figure 4: Mean square distances $E\left[|X(t, \omega)|^{2}\right]$ vs. time on the logarithmic scale with robust fit showing sub-diffusive scaling $O\left(t^{p}\right), p=0.483,0.673$ at $\theta=0.1$ (left) and 0.4 (right).

\section{Numerical Methods}

Recall that we are solving the following evolution problem over the unit square domain $\Omega=[0,1] \times[0,1]$ with periodic boundary condition and a constant initial condition:

$$
\left\{\begin{array}{l}
w_{t}=\epsilon \triangle w+(2 \epsilon \lambda \vec{e}+B) \cdot \nabla w-\left(C_{M}-\epsilon \lambda^{2}-\lambda \vec{e} \cdot B-\frac{1}{\tau} f^{\prime}(0)\right) w \\
w(0, y, t)=w(1, y, t) ; w(x, 0, t)=w(x, 1, t), \\
w(x, y, 0)=1 .
\end{array}\right.
$$

In the paper, we use standard notation of Sobolev spaces.

- $L^{2}(\Omega)$ stands for the Hilbert space of square integrable functions on $\Omega$.

- $H^{1}(\Omega)=\left\{v \in L^{2}(\Omega): \nabla v \in L^{2}(\Omega)\right\}$. 

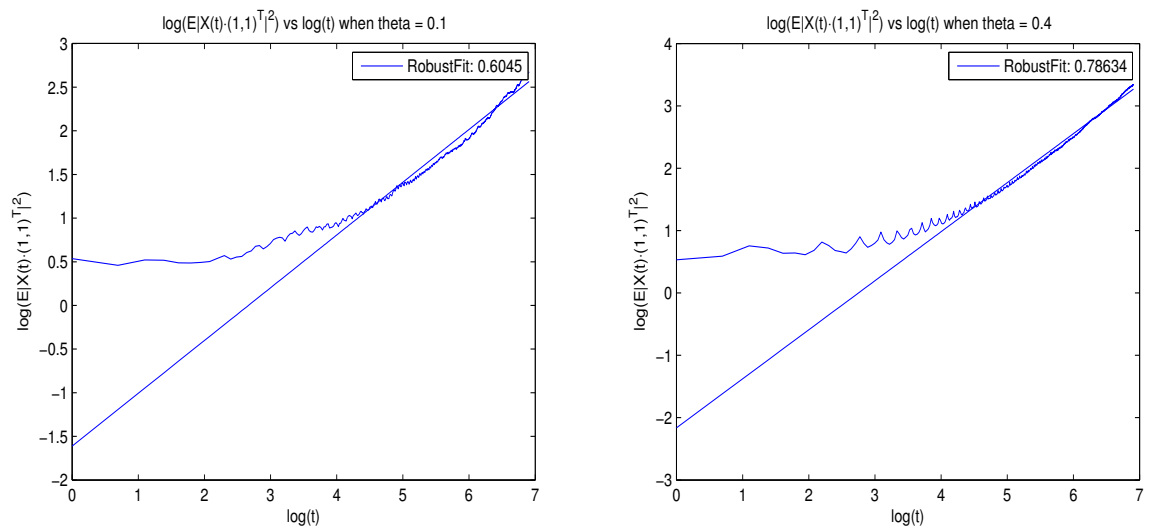

Figure 5: Projected mean square distances $E\left[(X(t, \omega) \cdot(1,1))^{2}\right]$ vs. time on the logarithmic scale with robust fit showing sub-diffusive scaling $O\left(t^{q}\right), q=$ $0.605,0.786$ at $\theta=0.1$ (left) and 0.4 (right).

- $H_{0}^{1}(\Omega)=\left\{u \in H^{1}(\Omega),\left.u\right|_{\partial \Omega}=0\right\}$

Numerical approximation of advection-dominated problems is a topic of independent interest. We shall use the Edge-Averaged Finite Element (EAFE) method [32] due to the nice discrete maximum principle obeyed by EAFE and the corresponding fast multigrid solvers. On the other hand, semi-implicit pseudo-spectral methods are a class of highly accurate numerical methods for solving partial differential equations. In practice, semi-implicit pseudo-spectral method has excellent error reduction properties with the so-called "exponential convergence" being the fastest possible as long as the solution is smooth. In this section, we briefly review these two methods used in our simulation.

\subsection{EAFE Method}

We first decompose the domain $\Omega$ into a triangulation $\mathcal{T}$ which is a set of triangles such that

$$
\cup_{\tau \in \mathcal{T}} \tau=\bar{\Omega} \quad \text { and } \quad \stackrel{\circ}{\tau}_{i} \cap \stackrel{\circ}{\tau}_{j}=\emptyset . \quad \text { for } \quad i \neq j
$$

For the unit square, we simply use the uniform mesh obtained by setting length of each triangle $h$ and divide each small square to 2 triangles by the diagonal in $(1,1)$ direction.

We describe the EAFE method using a simple advection-diffusion equation

$$
\begin{cases}-\nabla \cdot(\nabla u+\beta(x) u)=f(x), & x \in \Omega \\ u=0 & x \in \partial \Omega\end{cases}
$$

Associated with each $\mathcal{T}_{h}$, let $V_{h} \subset H_{0}^{1}(\Omega)$ be the piecewise linear finite element space. 
Given any edge $E$ in the triangulation, we introduce a function $\phi_{E}$ defined locally on $E$ (up to an arbitrary constant) by the relation:

$$
\frac{\partial \phi_{E}}{\partial t_{E}}=\frac{1}{\left|t_{E}\right|} \epsilon^{-1}\left(\beta \cdot t_{E}\right)
$$

where $E$ is the edge connecting two vertices $q_{i}$ and $q_{j}$, and $t_{E}$ is the vector such that $t_{E}=q_{i}-q_{j}$.

The EAFE formulation of the problem (3.3) is: Find $u_{h} \in V_{h}$ such that

$$
a_{h}\left(u_{h}, v_{h}\right)=f\left(v_{h}\right) \quad \text { for any } v_{h} \in V_{h}
$$

where

$$
a_{h}\left(u_{h}, v_{h}\right)=\sum_{T \in \mathcal{T}_{h}}\left\{\sum_{E \subset T} \frac{\omega_{E}^{T}}{\left|t_{E}\right|} \int_{E} e^{\phi_{E}} J(u) \cdot t_{E} d s \delta_{E} v_{h}\right\}
$$

with

$$
\left\{\begin{array}{l}
\omega_{E}=\frac{1}{2} \sum_{E \subset T} \cot \theta_{E}^{T} \geq 0 \\
J(u)=\epsilon \nabla u+\beta u
\end{array}\right.
$$

where $\theta_{E}^{T}$ is the angle opposite to the sharing common edge $\mathrm{E}$ in the triangle $T$ and $\delta_{E}$ is related to the tangential derivative along $E$.

The authors of [32] show that if the triangulation is a so-called Delaunay triangulation, i.e., the summation of two angles of an interior edge is less than or equal to $\pi$, the matrix of the linear system generated by EAFE would be an M-matrix with nonnegative row sum. The detail of the proof can be found in [32].

When applied to equation (3.1), notice that $\vec{B}$ is divergence free, the operator can be written in the divergence form of (3.3) and the reaction term is always positive. We apply the mass lumping method [27] so that the discretization of the reaction term makes positive contribution to the diagonal. Therefore the M-matrix property and consequently the discrete maximum principle still holds.

Remark 1 We have tested the Box method [5], EAFE method [32] and streamline diffusion method [11] on our problem. The accuracy of EAFE scheme is better than Box method, but lower than the streamline diffusion method. We chose EAFE instead of streamline diffusion scheme because the matrix of linear system generated by EAFE is M-matrix. The M-matrix not only preserves the discrete maximum principle (so that the numerical solution stays between 0 and 1) but also benefits from the fast solvers.

To accelerate the speed of computation, Algebraic Multi-Grid (AMG) solver is used to solve the linear algebraic equation arising from each time step. When the matrix is an M-matrix, the corresponding AMG solver is proven to be efficient. Specifically for the advection-diffusion equations, a multigrid method preserving the M-matrix property in coarse level is developed in [13]. Among many AMG software packages, we use AGMG (aggregation-based algebraic multigrid method) package [20]. The numerical test shows that AMG solver is 8 times faster than the default solver in MATLAB. We implemented the schemes based on the MATLAB ${ }^{\complement}$ software package $i$ FEM [17]. 


\subsection{Semi-Implicit Pseudo-Spectral Method}

To describe the semi-implicit pseudo-spectral method, we consider the problem:

$$
\begin{aligned}
& u_{t}=\epsilon \Delta u+\vec{B}(x, y, t) \cdot \nabla u+C u \\
& u(x, y, 0)=1 ; u(0, y, t)=u(1, y, t) \\
& u(x, 0, t)=u(x, 1, t)
\end{aligned}
$$

The derivatives of solutions are computed via $\nabla u=\mathcal{F}^{-1}(i \vec{k} \mathcal{F}(u))$ and $\triangle u=$ $\mathcal{F}^{-1}\left((i \vec{k})^{2} \mathcal{F}(u)\right)$. Here $\mathcal{F}$ is the Fourier transformation and $\vec{k}$ represents the wave numbers.

The semi-implicit pseudo-spectral scheme with semi-implicit scheme is that diffusion term uses implicit scheme and advection term uses half step lagged explicit scheme:

$$
\frac{\hat{U}^{n+1}-\hat{U}^{n}}{\Delta t}=-\epsilon \vec{k}^{2} \hat{U}^{n+1}+\mathcal{F}\left(\vec{B} \cdot \mathcal{F}^{-1}\left(i \vec{k} \hat{U}^{n}\right)\right)+C \hat{U}^{n}
$$

Here the discretization on the spatial domain is $N \times N$ and $\hat{U}^{n}$ is the frequency of $u$ at time step $n$. Semi-implicit pseudo-spectral method has higher order accuracy than the finite element methods. However, its time step size $\Delta t$ depends on the small diffusion parameter $\epsilon$. Therefore it is more costly to reach the large time solution.

\subsection{Stability Analysis}

The semi-implicit pseudo-spectral method is not so efficient when diffusion parameter $\epsilon$ goes to zero. Here we compare the upwinding finite element method and semi-implicit pseudo-spectral method. The analysis suggests a hybrid algorithm combining the EAFE finite element method and the semi-implicit pseudospectral method.

\subsubsection{Upwinding Finite element method}

The stability condition of EAFE method is equivalent to that of an upwinding scheme. To simplify analysis, the advection term is taken as one-dimensional and the reaction term is ignored. The proto-type equation is

$$
u_{t}=\epsilon u_{x x}+b u_{x}
$$

The upwinding Crank-Nicolson scheme is

$$
\left\{\begin{array}{rlrl}
\frac{U_{j}^{n+1}-U_{j}^{n}}{\Delta t} & =\epsilon\left(\frac{U_{j+1}^{n}+U_{j-1}^{n}-2 U_{j}^{n}}{2}+\frac{U_{j+1}^{n+1}+U_{j-1}^{n+1}-2 U_{j}^{n+1}}{2}\right) / \Delta x^{2} \\
& +\frac{b}{\Delta x}\left(\frac{U_{j+1}^{n+1}-U_{j}^{n+1}}{2}+\frac{U_{j+1}^{n}-U_{j}^{n}}{2}\right) \quad \text { if } b \geq 0 \\
\frac{U_{j}^{n+1}-U_{j}^{n}}{\Delta t} & =\epsilon\left(\frac{U_{j+1}^{n}+U_{j-1}^{n}-2 U_{j}^{n}}{2}+\frac{U_{j+1}^{n+1}+U_{j-1}^{n+1}-2 U_{j}^{n+1}}{2}\right) / \Delta x^{2} \\
& +\frac{b}{\Delta x}\left(\frac{U_{j}^{n+1}-U_{j-1}^{n+1}}{2}+\frac{U_{j}^{n}-U_{j-1}^{n}}{2}\right) & \text { if } b<0
\end{array}\right.
$$


Denoting

$$
b^{+}=\max (b, 0), \quad b^{-}=\min (b, 0),
$$

we carry out the von Neumann analysis to obtain the stability condition. Upon substitution $U_{j}^{n}=\rho^{n} e^{i j \theta},(3.10)$ becomes:

$$
\frac{\rho-1}{\Delta t}=\frac{\epsilon(\rho+1)(\cos (\theta)-1)}{\Delta x^{2}}+\frac{|b|(1+\rho)(\cos (\theta)-1+i \sin (\theta))}{2 \Delta x}
$$

Let $\mu=\frac{\epsilon \Delta t}{\Delta x^{2}}$ and CFL number $C=\frac{|b| \Delta t}{\Delta x}$. The Peclet number

$$
P e=\frac{\text { advection }}{\text { diffusion }}=\frac{|b| \Delta x}{\epsilon}=C / \mu \text {. }
$$

Equation (3.11) simplifies to:

$$
\left(1+\left(\mu+\frac{C}{2}\right)(1-\cos \theta)-\frac{i C \sin \theta}{2}\right) \rho=1-\left(\mu+\frac{C}{2}\right)(1-\cos \theta)+\frac{i C \sin \theta}{2}
$$

The stable condition is:

$$
\rho \leq 1 \Longleftrightarrow \frac{1-\left(\mu+\frac{C}{2}\right)(1-\cos \theta)}{1+\left(\mu+\frac{C}{2}\right)(1-\cos \theta)} \leq 1
$$

and the right inequality is always true because $\left(\mu+\frac{C}{2}\right)(1-\cos \theta) \geq 0$.

So the upwinding Crank-Nicolson is unconditional stable.

\subsubsection{Semi-Implicit Pseudo-Spectral Method}

Consider the semi-implicit pseudo-spectral method on the proto-type equation (3.9) again: we have shown before:

$$
\frac{U^{n+1}-U^{n}}{\triangle t}=-\epsilon \vec{k}^{2} U^{n+1}+b \mathcal{F}^{-1}\left(i \vec{k} \mathcal{F} U^{n}\right)
$$

and $\vec{k}=(\cdots,-3,-2,-1,0,1,2,3, \cdots)$

To prove the stability of the semi-implicit pseudo-spectral method with semiimplicit scheme, we assume:

$$
u(x, t)=\sum_{k=-\infty}^{\infty} \hat{u}_{k}(t) e^{i k x}
$$

and we denote the projection operator on the discrete level:

$$
U=\left(P_{N}\right) u(x, t)=\sum_{-\frac{N}{2}<k \leq \frac{N}{2}} \hat{u}_{k}(t) e^{i k x}
$$


If we plug (3.15) and (3.16) into (3.14), we have that the k-th component of the vector, which should be the corresponding coefficient of $e^{i k x}$ :

$$
\frac{\hat{U}_{k}^{n+1}-\hat{U}_{k}^{n}}{\triangle t}=-\epsilon k^{2} \hat{U}_{k}^{n+1}+i k b \hat{U}_{k}^{n} .
$$

for $k=1,2,3$.

From (3.17), we obtain

$$
\left|\hat{u}_{k}^{n+1}\right|=\left|\frac{1+i k b \triangle t}{1+\epsilon k^{2} \triangle t} \hat{u}_{k}^{n}\right| \leq \frac{|1+i k b \triangle t|}{\left|1+\epsilon k^{2} \triangle t\right|}\left|\hat{u}_{k}^{n}\right|
$$

So $U^{n}$ in (3.14) is stable if and only if:

$$
\left|\frac{1+i k b \triangle t}{1+\epsilon k^{2} \triangle t}\right| \leq 1 \quad \forall k \in\left\{-\frac{N}{2}<k \leq \frac{N}{2}\right\}
$$

which is equivalent to

$$
\triangle t \leq \frac{2 \epsilon}{b^{2}-\epsilon^{2} k^{2}} \quad \forall k \in\left\{-\frac{N}{2}<k \leq \frac{N}{2}, k \neq 0, b^{2}-\epsilon^{2} k^{2}>0\right\}
$$

So if the equation is advection dominant, then at least $k=1$ satisfies

$$
-\frac{N}{2}<k \leq \frac{N}{2}, \quad k \neq 0, \quad b^{2}-\epsilon^{2} k^{2}>0
$$

and the stable condition would be $\triangle t \leq \frac{2 \epsilon}{b^{2}-\epsilon^{2}}$. When $\epsilon \ll 1$, the time step is in the order of $\mathcal{O}(\epsilon)$ which is very restrictive.

\section{Numerical Results on Residual Speeds}

\subsection{Steady Flow}

In order to show that the numerical result of the scheme above is convincing, we validate our scheme by solving the steady flow problem in [26] and report a result consistent with existing one in the literature. And the stopping criterion with respect to time is letting the relative error of the average of numerical solution in every 100 time steps less than $5 \times 10^{-4}$. Just to clarify the possible confusion, the stopping criterion here is used to calculate the principle Lyapunov exponent of the parabolic equation (1.5). After obtaining $\mu(\lambda)$, a minimal problem needs to be solved on a different criterion and that will be discussed at the end of section 4 .

The flow function is $\left(-100 \sin (2 \pi x) \cos (2 \pi y), 100 \cos (2 \pi x) \sin (2 \pi y)^{T}\right.$ in this section. In Fig. 6, the relation between $\epsilon$ and $c^{*}$ is obtained from the method proposed in the paper. 

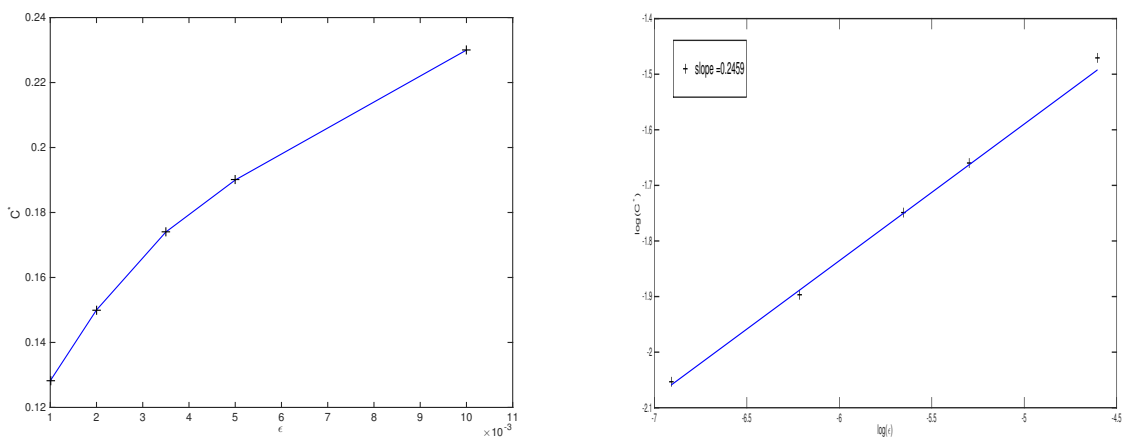

Figure 6: Relation between $\epsilon$ and $c^{*}$ in steady cellular flow(Left). The slope of the relation between $\log (\epsilon)$ and $\log \left(c^{*}\right)$ (Right) shows that $c^{*}=\mathcal{O}\left(\epsilon^{p}\right), p=$ $0.2459 \approx 0.25$, is consistent with the result in $[21,26]$

\subsection{Unsteady Flow}

For each given $\lambda$, we compute the Lyapunov exponent of (1.4) by finding the long time quasi-stationary solution $u$ and applying formula (1.6). A quasi-stationary solution with $\lambda=1$ is shown in the left plot of Fig. 7. The layer structure of the numerical solution moves along the $y=x$ direction and oscillates between the two neighboring invariant manifolds (lines of unit slope). The right plot of Fig. 7 shows the power spectrum of the auxiliary field (a genearalized eigenfunction) $w$ (right) for the unsteady flow (2.2). We observe that the energy decreases towards high frequency, with a linear decay (scaling range) in the intermediate region of wave numbers.

After calculating $\mu$ by the algorithm discussed in (1.6), the minimization problem (1.3) is solved by the Golden-section method [4]. Golden-section method is a method to find the minimal point of a particular function called unimodal function. The idea of Golden-section method is by successively narrowing down the range of searching interval. A common definition of unimodal function is for some value $m$, it is monotonically decreasing when $x<m$ and monotonically increasing when $x \geq m$. In [19], the authors show that the function $\frac{\mu(\lambda)}{\lambda}$ is indeed a unimodal function which is strictly convex with respect to $\lambda$, decreasing over interval $\left[0, \lambda^{*}\right]$ and strictly increasing over interval $\left[\lambda^{*}, \infty\right]$, with $\lambda^{*}$ the unique minimal point of $\frac{\mu(\lambda)}{\lambda}$. Notice that the popular Newton's method is not applicable since $\mu^{\prime}(\lambda)$ is not known.

For time periodic cellular flow (1.1), EAFE method and semi-implicit pseudospectral method are combined together to obtain the result in Fig. 8. The initial searching interval is $[0,1000]$. EAFE is used first to narrow down the searching interval to an neighborhood of $\lambda^{*}$ with length 2 . The space size and time step is $h=2^{-10}$ and $d t=10^{-7}$, respectively. Since the minimal diffusion parameter $\epsilon=\mathcal{O}\left(10^{-3}\right)$ and amplitude of advection term is $\mathcal{O}\left(10^{3}\right)$, the choice of $d t=10^{-7}$ leads to numerical method stable. The spectral method with space node $N=2^{9}$ 

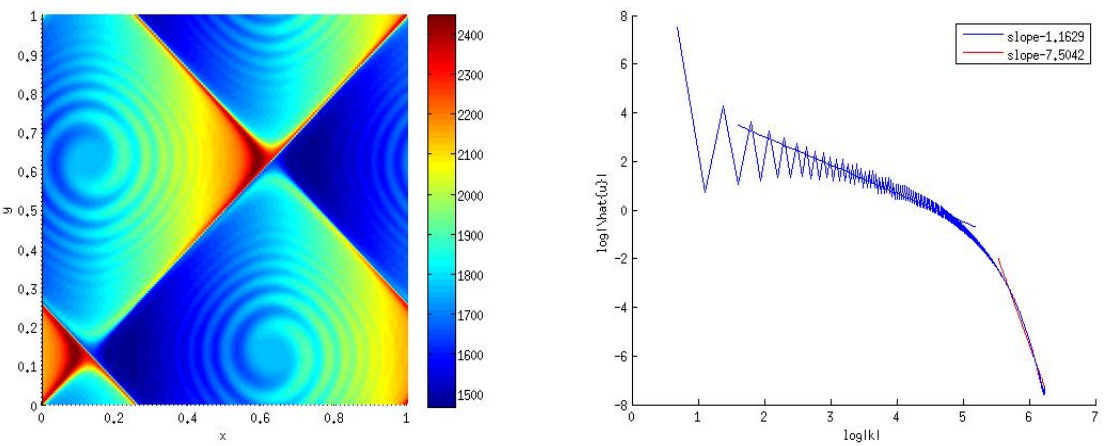

Figure 7: A quasi-stationary solution (left) at time $t=2, \epsilon=10^{-3},|B|=500$, and $\lambda=1$. The number of space nodes $N=2^{9}$ in each direction and time step $d t=10^{-8}$. The power spectrum of the auxiliary field $w$ (right) for the unsteady flow (2.2) shows that the energy decreases towards high frequency, with a scaling range (slope $=-1.1629$ ) in the intermediate region of wave numbers.

in each direction and time step $d t=10^{-8}$ is used to obtain the minimal point when the length of searching interval is less than 2. The stopping criterion of searching algorithm is that the difference of two consecutive values of $\frac{\mu(\lambda)}{\lambda}$ is less than $5 \times 10^{-3}$. The benefit of using two methods together is that we can find the minimal point faster without the sacrifice of the accuracy. When we use Golden-section method to solve the minimal problem, each iteration will reduce the length of interval by golden raio 0.618 . For each $\lambda$, about 14 times of iteration is done by EAFE method and this reduce the size of interval to $1 / 1000$ of the original interval. And the semi-implicit pseudo-spectral method is applied to the refined interval.

In Fig. 8, we plot $c^{*}$ as a function of $\epsilon$ for three values of parameter $\theta=$ $10^{-2}, 10^{-1}, 1$. Recall that $\theta$ is the perturbation parameter which determines how close the flow is to the steady cellular flow; see (1.1). From Fig. 8, we observe that for all three positive values of $\theta, c^{*}(\epsilon)=O(1)$ as $\epsilon \downarrow 0$. In contrast, it is well known that $c^{*}(\epsilon)=O\left(\epsilon^{1 / 4}\right)$ in steady cellular flows [21]. The presence of chaotic trajectories in time periodic cellular flows contributed to this phenomenon. They are much more mobile and far reaching than their counterparts of steady cellular flows. Fig. 9 shows the persistence of residual front speeds under grid refinement at $\theta=1$ and the close proximity of the data points between those at grid sizes of $N=2^{8}$ and $N=2^{9}$ validates the convergence of the results numerically.

\section{Concluding Remarks}

We have studied the KPP front speed asymptotics computationally in time periodic cellular flows with chaotic streamlines in the small molecular diffusivity limit. The chaotic streamlines statistically resemble a sub-diffusion process and 


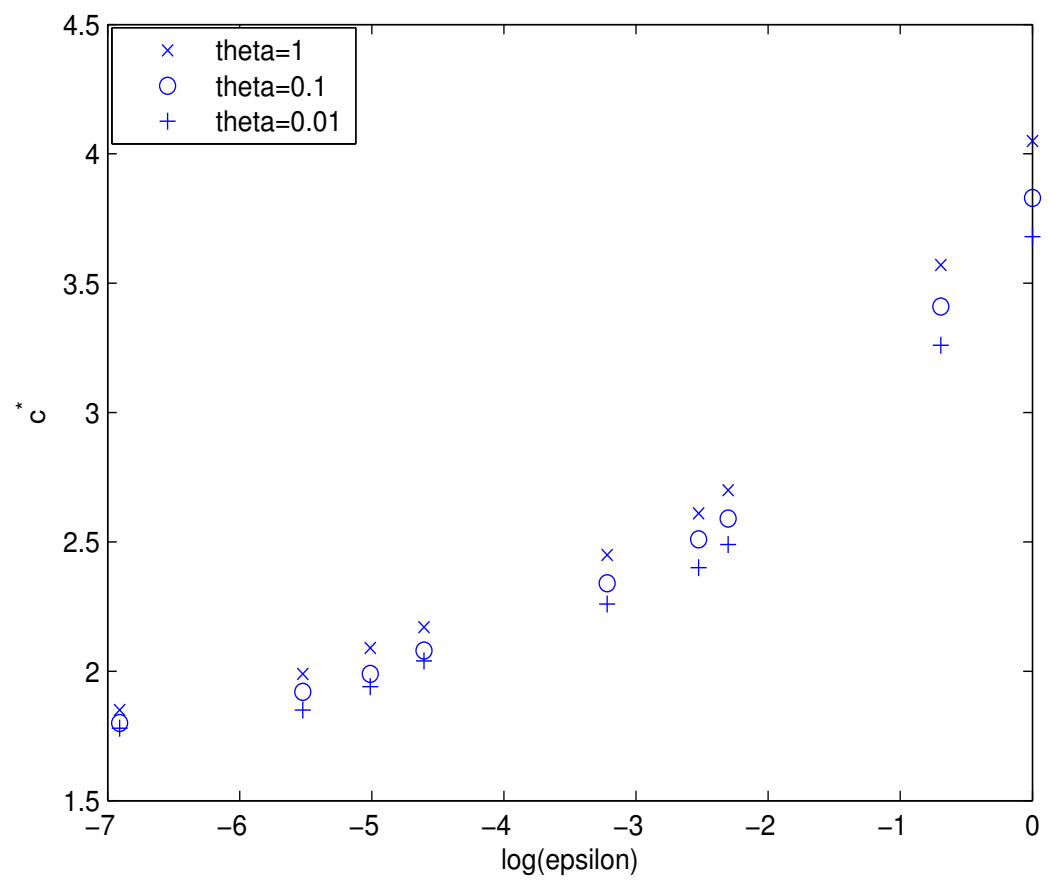

Figure 8: Residual KPP front speeds in the small diffusion limit $\epsilon \downarrow 0$.

enhance the KPP front speed $c^{*}$ significantly in the sense that $c^{*}$ has a positive limit as molecular diffusivity tends to zero. Such residual transport phenomenon is absent in steady cellular flows with ordered streamlines. To facilitate effective computation in the advection dominated regime, we combined an upwinding finite element methods with semi-implicit pseudo-spectral method for computing the principle eigenvalue of a time periodic parabolic operator with small diffusion and for a subsequent minimization. In future work, we plan to study residual KPP front speeds in three space dimensional chaotic flows.

\section{Acknowledgements}

The work was partially supported by NSF grant DMS-1211179. We thank Tyler McMillen for helpful conversations and visualization of chaotic dynamical systems. L.C was also supported by NSF grant DMS-1115961, DMS-1418732 and DOE prime award \# DE-SC0006903. 


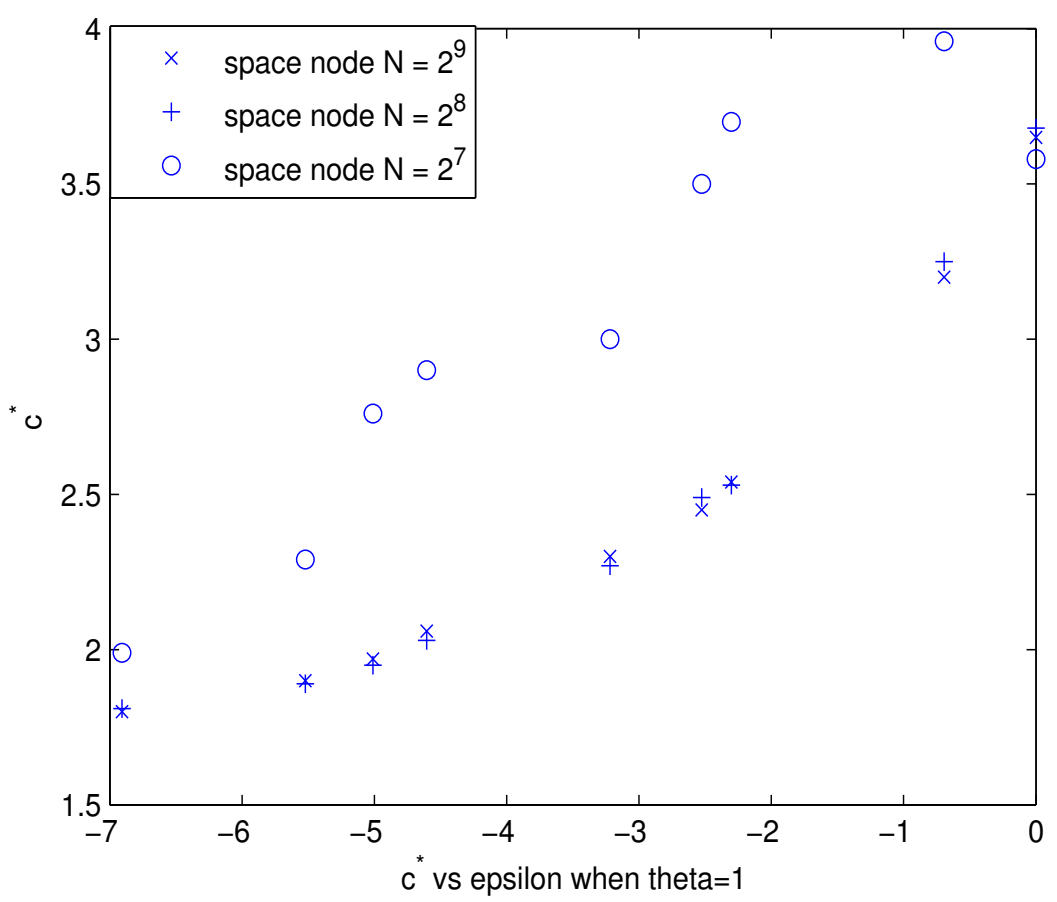

Figure 9: Residual KPP front speeds at $\theta=1$ under grid refinement.

\section{References}

[1] E. Abraham, et al., Importance of stirring in the development of an ironfertilized phytoplanton bloom, Nature 407 (2000), pp. 727-730.

[2] M. Abel, M. Cencini, D. Vergni, A. Vulpiani, Front speed enhancement in cellular flows, Chaos 12 (2002), pp. 481-488.

[3] B. Audoly, H. Berestycki, Y. Pomeau, Réaction diffusion en écoulement stationnaire rapide, C.R. Acad. Sci. Paris, Série IIb, 328 (2000), pp. 255-262.

[4] R. Brent, "Algorithms for Minimization Without Derivatives", Prentice Hall, 1973, DFMIN module http://gams.nist.gov/serve.cgi/Module/NMS/DFMIN/5671/.

[5] R. Bank, J. Burger, W. Fichtner, R. Smith, Some upwinding techniques for finite element approximations of convection diffusion equations, Numer. Math., 58 (1990), pp. 185-202.

[6] L. Biferale, A. Cristini, M. Vergassola, A. Vulpiani, Eddy diffusivities in scalar transport, Physics Fluids 7(11), 1995, pp. 2725-2734. 
[7] A. Brooks, T. Hughes, Streamline upwind/Petrov-Galerkin formulations for convection dominated flows with particular emphasis on the incompressible Navier-Stokes equations. Computer methods in applied mechanics and engineering 32(1), 1982, pp. 199-259.

[8] R. Camassa, S. Wiggins, Chaotic advection in a Rayleigh-Bénard flow, Physical Review A, 43(2), 1990, pp. 774-797.

[9] S. Childress, A. Soward, Scalar transport and alpha-effect for a family of cat's eye flows, J. Fluid Mech. 205 (1989), pp. 99-133.

[10] J. Cooper, J. Butcher, An Iterative Scheme for Implicit Runge-Kutta Methods, IMA J. Numer. Anal. 3, pp. 127-140, 1983.

[11] H. Elman, D. Silvester, A. Wathen, "Finite Elements and Fast Iterative Solvers with Applications in Incompressible Fluid Dynamics", Oxford University Press, 2005.

[12] A. Fannjiang, G. Papanicolaou, Convection enhanced diffusion for periodic flows, SIAM J. Applied Math, 54(2), 1994, pp. 333-408.

[13] H. Kim, J. Xu, and L. Zikatanov, A multigrid method based on graph matching for convection-diffusion equations, Numer. Linear Algebra Appl., (2003), pp. 181 - 195. More details are at: http://epubs.siam.org/doi/ref/10.1137/060659545

[14] Y. Gorb, D. Nam, and A. Novikov, Numerical simulations of diffusion in cellular flows at high Peclet numbers, DCDS-B, vol. 51(1), 2011, pp. $75-92$.

[15] S. Heinze, Diffusion-advection in cellular flows with large Peclet numbers, Archive Rational Mech. Analysis, 168(4), 2003, pp. 329-342.

[16] Y. Liu, J. Xin, Y. Yu, A Numerical Study of Turbulent Flame Speeds of Curvature and Strain G-equations in Cellular Flows, Physica D, 243(1), pp. 20-31, 2013.

[17] L. Chen. iFEM: An Integrated Finite Element Methods Package in MATLAB. Technical Report, University of California at Irvine, 2009.

[18] J. Nolen, J. Xin, Asymptotic Spreading of KPP Reactive Fronts in Incompressible Space-Time Random Flows, Ann Inst. H. Poincaré, Analyse Non Lineaire, 26(3), 2009, pp. 815-839.

[19] J. Nolen, M. Rudd, J. Xin, Existence of KPP fronts in spatiallytemporally periodic advection and variational principle for propagation speeds, Dynamics of PDE, 2(1), pp. 1-24, 2005.

[20] Y. Notay, AGMG software and documentation; see http://homepages.ulb.ac.be/ ynotay/AGMG 
[21] A. Novikov, L. Ryzhik, Boundary layers and KPP fronts in a cellular flow, Arch. Ration. Mech. Anal., 184(1), 2007, pp. 23 - 48.

[22] M. Paoletti, T. Solomon, Experimental studies of front propagation and mode-locking in an advection-reaction-diffusion system, Europhys. Lett. 69 (2005), pp. 819-825.

[23] P. Ronney, Some Open Issues in Premixed Turbulent Combustion, Modeling in Combustion Science (J. D. Buckmaster and T. Takeno, Eds.), Lecture Notes In Physics, Vol. 449, Springer-Verlag, Berlin, 1995, pp. 3-22.

[24] L. Ryzhik, A. Zlatos, KPP pulsating front speed-up by flows, Commun. Math. Sci. 5 (2007), pp. 575-593.

[25] W. van Saarloos, Front propagation into unstable states, Physics Reports 386(2), 2003, pp. 29-222.

[26] L. Shen, A. Zhou, J. Xin, Finite Element Computation of KPP Front Speeds in Cellular and Cat's Eye Flows, Journal of Scientific Computing, 55(2), 2013, pp. 455-470.

[27] P. Tong, T.H.H. Pian, L.L. Bucciarelli, Mode shapes and frequencies by finite element method using consistent and lumped masses, Comput. Struct. 1 (1971), pp. 623-638.

[28] F. Williams, "Combustion Theory", Benjamin-Cummings, Menlo Park, 1985.

[29] J. Xin, "An Introduction to Fronts in Random Media", Surveys and Tutorials in Applied Math Sciences, Vol. 5, Springer, 2009.

[30] J. Xin, Y. Yu, Front Quenching in G-equation Model Induced by Straining of Cellular Flow, Arch. Rational Mechanics Analysis, 214 (2014), pp. 1-34..

[31] J. Xin, Y. Yu, Asymptotic growth rates and strong bending of turbulent flame speeds of G-equation in steady two dimensional incompressible periodic flows, SIAM J. Math Analysis, 46(4), 2444-2467, 2014.

[32] J. Xu, L. Zikatanov, A monotone finite element scheme for convection diffusion equations, Math. Comp., 68 (1999), pp. 1429-1446. 